\title{
The Use of a Bone-Anchored Device as a Hard- Wired Conduit for Transmitting EMG Signals from Implanted Muscle Electrodes
}

\author{
Y. Al-Ajam*, H. Lancashire, C. Pendegrass, N. Kang, R. P. Dowling, S. J. G. Taylor, and G. Blunn
}

\begin{abstract}
The use of a bone-anchored device to transmit electrical signals from internalised muscle electrodes was studied in a sheep model. The bone-anchored device was used as a conduit for the passage of a wire connecting an internal epimysial electrode to an external signal-recording device. The boneanchored device was inserted into an intact tibia and the electrode attached to the adjacent $M$. peroneus tertius. 'Physiological' signals with low signal to noise ratios were successfully obtained over a 12 -week period by walking the sheep on a treadmill. Reliable transmission of multiple muscle signals across the skin barrier is essential for providing intuitive, biomimetic upper-limb prostheses. This technology has the potential to provide a better functional and reliable solution for upper-limb amputee rehabilitation: attachment and control.
\end{abstract}

Index Terms - Prosthetic limbs, implantable myoelectrodes, bone-anchored device, myoelectric control.

\section{INTRODUCTION}

$\mathrm{T}$ HERE are 94,000 estimated upper limb amputees in Europe [1] and a further 41,000 in the United States [2]. Upper limb loss results in significant physical and psychological morbidity with the majority of cases occurring due to trauma in young patients.

Prostheses have remained the mainstay for significant upper limb loss rehabilitation, however they pose 2 main challenges;

Manuscript received July $16^{\text {th }} 2012$. This work was supported in part by the Royal College of Surgeons of England Surgical Research Fellowship, Restoration of Appearance and Function Trust (RAFT), Rosetrees Trust, EPSRC.

A preliminary version of this work was presented at the $57^{\text {th }}$ Annual Meeting of the Plastic Surgery Research Council, Ann Arbor, Michigan, USA, June 2012. Asterisk indicates corresponding author.

* Y Al-Ajam is with the Institute of Biomedical Engineering, University College London, Royal National Orthopaedic Hospital, Stanmore, HA7 4LP, UK. (phone: +44 208909 5304; fax: +44 208420 7392; e-mail: yazan.ajam@gmail.com).

H. Lancashire, C. Pendegrass, R. P. Dowling, S. J. G. Taylor, and G. Blunn are with the Institute of Biomedical Engineering, UCL, RNOH, Stanmore HA7 4LP, UK (email: henry.lancashire.10@ucl.ac.uk; c.pendegrass@ucl.ac.uk; robert.p.dowling@gmail.com; stephen.taylor@ucl.ac.uk; g.blunn@ucl.ac.uk).

N. Kang is with the Department of Plastic and Reconstructive Surgery, Royal Free Hospital, Pond Street, London, NW3 2 QG, UK (email norbertkang@btinternet.com). attachment of the prosthesis to the amputation stump and control of the end device.

Current prostheses are usually controlled using muscle signals from skin-surface electrodes, typically two, placed on antagonist muscles. These signals can be used directly, to drive a single degree of freedom (DoF), or to control an eventdriven finite-state map, where modes are selected by a rarely used signal (e.g. co-contraction or double-contraction) [3].

The inherent disadvantage of this system is that it is unintuitive, and because the prosthesis is controlled in a nonphysiological way, actions can only be performed in series, interposed by mode switching to change the DoF.

In a human hand there are $22 \mathrm{DoF}$ controlled by 38 muscles [4]. Upper limb prostheses require 38 actuators, each with a separate activating signal, to fully mirror hand motion. Therefore in order to improve hand control, an increase in the number of control channels is required. As the number of muscles adjacent to the humerus is limited, targeted muscle reinnervation (TMR) may provide a solution in above elbow amputees [5]. In this surgical technique peripheral nerves normally supplying the upper limb are rerouted to nerves innervating chest and torso muscles. Following nerve regeneration, patients are fitted with an array of surface electrodes to 'map out' the muscle areas responsible for specific hand actions. Patients are able to exert intuitive control of prostheses without the need for mode switching. However, this technique is less applicable in patients with trans-radial amputations. Furthermore, reliance on surface electrodes has its limitations, namely difficulty with placing the electrodes in a repeatable location, electrode lift-off, impedance variability due to skin moisture, and signal contamination from cross-talk caused by interference from deep or adjacent muscles [4], [6].

Although considerable advancements in improving intuitive control using pattern recognition have been made, reliability issues have precluded their use in commercial prostheses to date [7].

One solution to the problems associated with skin surface electrodes is placement of the electrodes directly on or in the muscle. This also allows targeting of individual muscles responsible for specific actions, increasing selectivity and reducing cross-talk. However, implantable electrodes pose a significant challenge with transcutaneous data transmission. 
Early designs (and some current cortical designs) use hardwired connections, these are either too fragile or too bulky, and can provide a route for infection, negating the advantage of implantable electrodes [8].

Although signal telemetry can be used, this increases the complexity of designs and can limit the number of channels. A recent electromyography (EMG) telemetry system by Weir et al. [6] transmitted a maximum of 32 channels of data at approximately 470 samples per second, with a low-pass corner of $<235 \mathrm{~Hz}$, this is sufficient for gross muscle movements, but high frequency EMG signals and some motor unit action potential spikes will not be detected [9].

Current prostheses, using suction cups, sleeves, sockets or harnesses result in sweating, chaffing, numbness, discomfort, heterotopic ossification and potentially serious skin infections. This combined with the low levels of dexterity provided by prosthetic limbs lead to low satisfaction and high rates of prosthesis abandonment [10].

For upper-limb amputees, osseointegration of a transcutaneous implant directly to the residual bone of the stump can provide a solution to the problems associated with prosthesis attachment and at the same time provide an engineered conduit for the hard wire coupling of electrodes and actuators. Clinical experience with an osseointegrated prosthesis for a proximal humeral amputee has shown this technique to be revolutionary [11]. Combining osseointegrated prostheses with a hard-wired connection will avoid the fragility of earlier hard-wired connections and the channel limitations of current telemetry devices.

The combination of bio-signal recording and direct skeletal attachment was first reported by Pitkin [12]. In a single, acute, recording session it was shown that EMG signals could be recorded from a sedated rabbit amputation model using silverplated wire electrodes.

The long-term reproducibility of signals with low noise from internal EMG electrodes is of considerable importance if this approach is to be used clinically. Our study aims to record EMG signals from a known muscle in an unsedated animal over a period of time and transfer these signals using a hardwired, bone-anchored prosthesis.

\section{METHODS}

\section{A. Bone-Anchored / Electrode Device Construction}

An osseointegrated transcutaneous implanted device was made from Ti-6Al-4V (Fig. 1.) as described by Pendegrass et al. [13]. A straight $2 \mathrm{~mm}$ hole was drilled from the top surface of the device, exiting immediately within the soft tissues above the region of the implant associated with osseointegration, this eliminated the need for an additional hole in the tibial cortex. A schematic of the device is shown in fig. 2 .

A bipolar epimysial electrode (Ardiem Medical Inc., PA, USA) was chosen. The electrode consisted of 2 circular Pt/Ir disks $\left(10.64 \mathrm{~mm}^{2}\right)$, with an interelectrode distance of $10.0 \mathrm{~mm}$, on a polyester reinforced silicone backing. The lead was 316

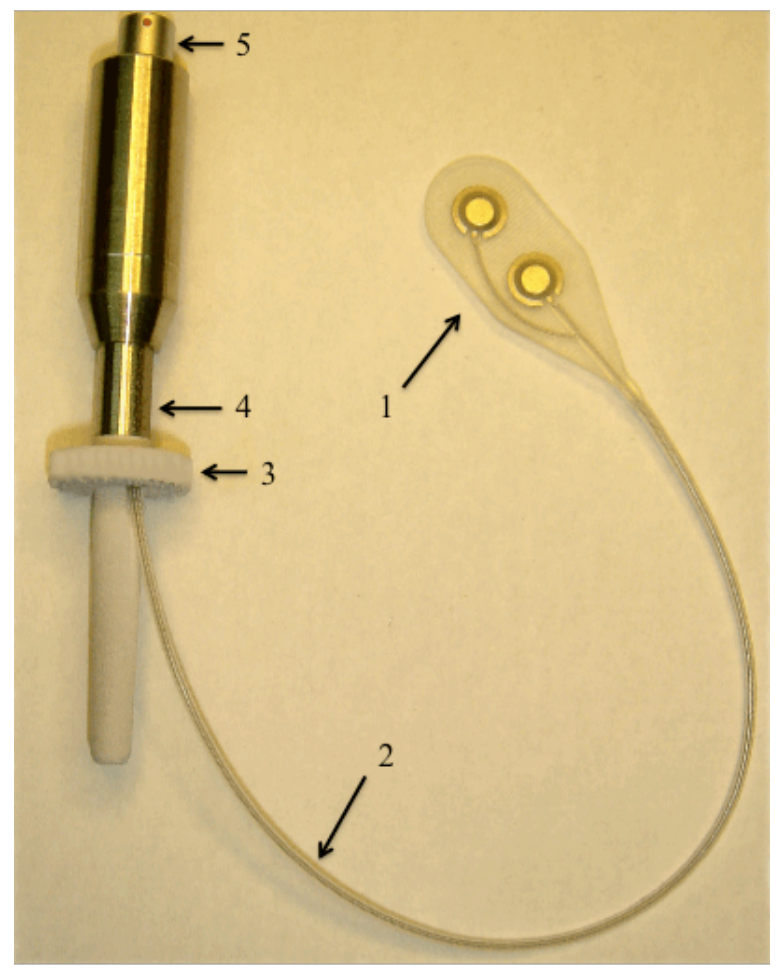

Fig. 1. Bone-anchored device with epimysial electrode (1). Electrode cable (2) is passed through a bore-hole immediately under the flange (3), through the core of the external part of the implant (4) and onto a 2-pin socket connector (5)

SS, 2-core coiled cable with fluoropolymer insulation encapsulated in silicone.

The cable for the epimysial electrode was passed through the implant and the flying leads were crimped to a 2-pin socket (LEMO UK Ltd, Worthing, U.K). The socket was held in place in a Ti-6Al-4V sleeve press fitted over the top of the device. The socket and sleeve were held in place using epoxy resin. The cable was secured in the $2 \mathrm{~mm}$ hole using medical grade silicone elastomer (NuSil Med3-4013, NuSil Technology LLC, CA, USA). The silicone was degassed under vacuum, injected using a syringe, and cured for 24 hours at $50^{\circ} \mathrm{C}$. Care was taken to ensure no uncured silicone leaked onto the HA coated flange or tapered stem. The implant

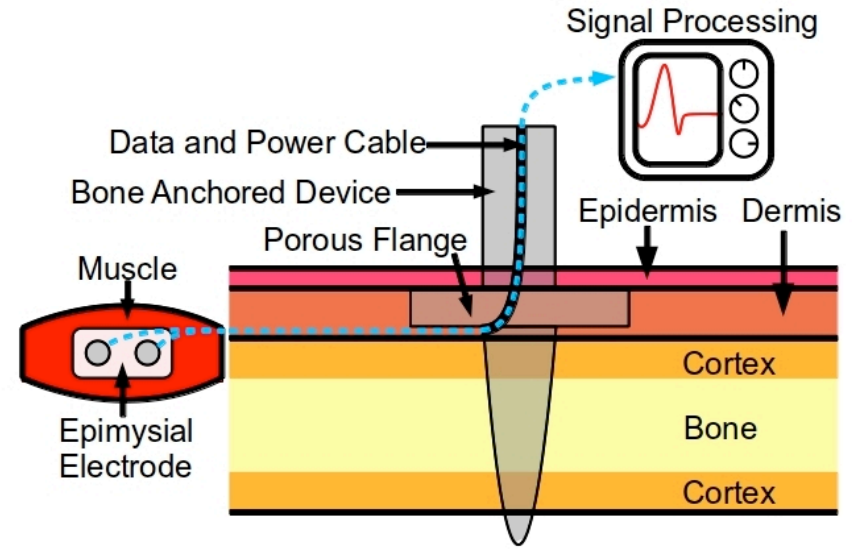

Fig. 2. Schematic of the device showing central bore hole in the boneanchored device exiting just underneath the flange. The wire runs between the bone and overlying soft tissue to the epimysial electrode, which is sutured onto the muscle. 
was sterilized using ethyl oxide gas.

\section{B. Surgical Technique}

One female sheep (Mule) was used for this study. All animal procedures were carried out in accordance with the Home Office Animals (Scientific Procedures) Act 1986. Under general anesthesia, an incision was made over the left tibia, $10 \mathrm{~cm}$ inferior to the knee joint and dissection made down to the bone (Fig. 3.). Soft tissue was stripped off the tibia, both cortices were drilled and reamed and the implant press fitted into the hole (trans-tibial insertion), allowing $2 \mathrm{~mm}$ between the flange and the underlying bone to accommodate
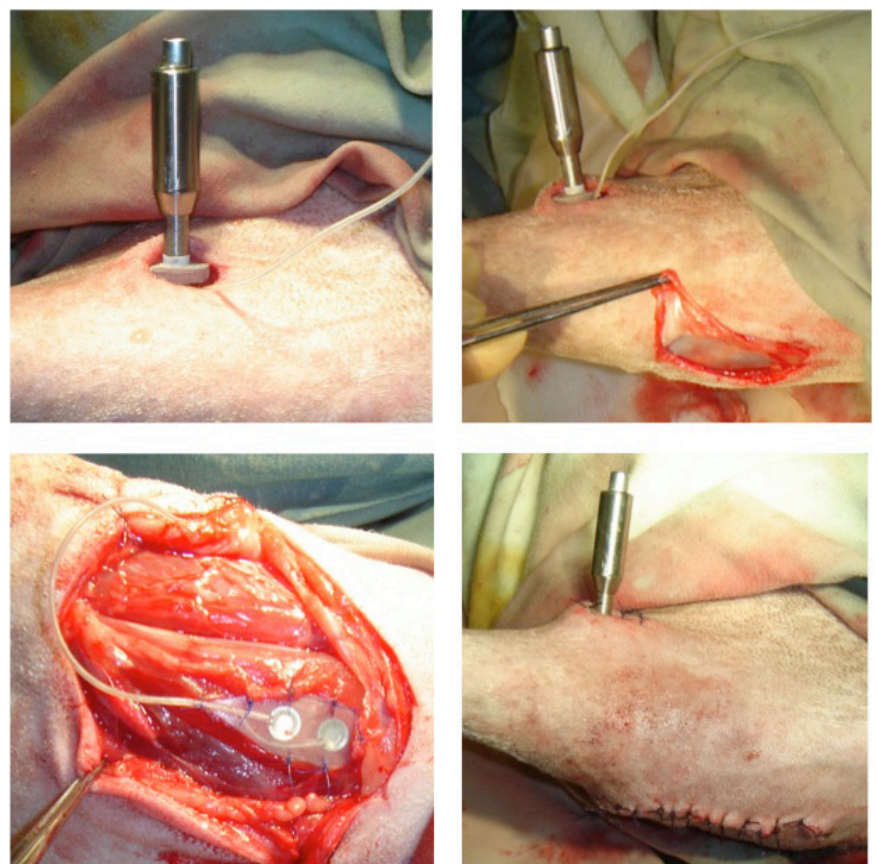

Fig. 3. Bone-anchored device inserted into tibia (top left). Separate incision made over $M$. peroneus tertius (top right), the electrode passed under the intervening skin bridge and sutured onto the muscle (bottom left). Wounds are closed in layers (bottom right).

the electrode wire.

A separate incision was made overlying the lateral compartment muscles of the left leg. M. peronius tertius was identified. A subcutaneous pocket was made under the intervening skin between the 2 incisions through which the electrode was passed. The electrode was secured into position on the muscle using 4-0 Prolene (non-absorbable) suture. The wounds were washed with saline and closed tension-free in layers with Vicryl (absorbable) suture (Ethicon Inc., NJ, USA). Mepitel (Mölnlycke Health Care Limited, Bedfordshire, UK), sterile gauze and a bandage were used as a post-operative dressing. The position of the implant was confirmed by plain radiographs (fig. 4.)

Antibiotics were given for 3 days post-operatively and fentanyl patches for one week. The animal was individually housed and the first EMG signals were obtained 24 hours post-operatively.

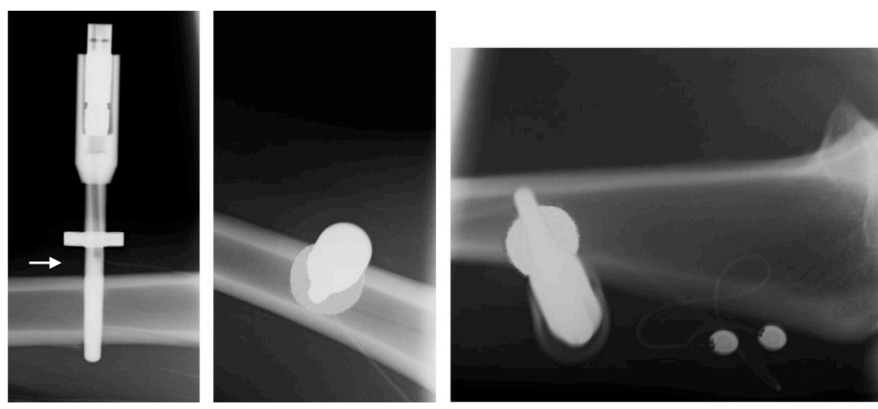

Fig. 4. Plain radiographs of the device taken post-operatively. Lateral view (left) showing gap between flange and bone to accommodate wire (arrow) anterior-posterior view (middle), oblique view showing epimysial wire and discs (right).

\section{EMG Recording}

On a weekly basis for 12 weeks, the animal underwent treadmill walking at $2 \mathrm{~km} / \mathrm{h}$ and signals captured using electromyogram (EMG) recording equipment (MP150 and EMG100, AcqKnowledge software, BIOPAC Systems, Inc., CA, USA) (Fig. 5.). EMG signals were recorded (at 500x gain, 1000 samples per second (sps), $100 \mathrm{~Hz}$ to $500 \mathrm{~Hz}$ band-pass). The electrode was accessed by attaching the lead from a recording device to the external part of the implant (fig. 1(5).) using a plug and socket interface.

Signals from overlying surface electrodes were used for comparison on week 2 . The skin was shaved and cleaned with alcohol and allowed to dry. Surface electrodes (Vermed Inc., VT, USA) were placed on the skin directly overlying $M$.

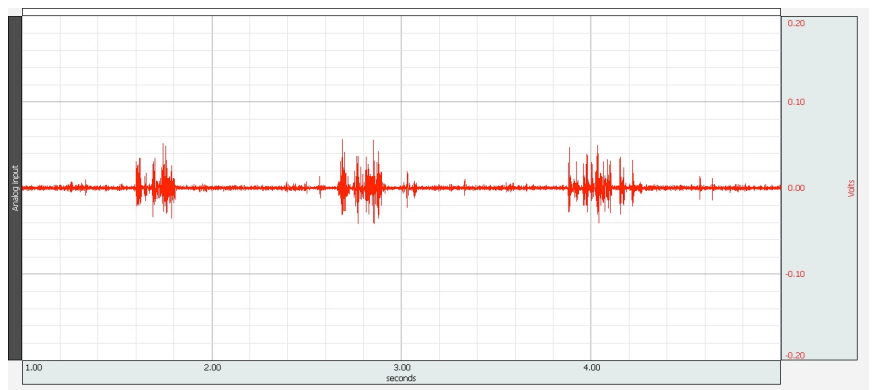

Fig. 5. Typical epimysial EMG recording obtained during treadmill walking. 3 distinct EMGs can be seen. Timeline on $x$-axis in seconds (large divisions).

peronius tertius at an inter-electrode distance of $20 \mathrm{~mm}$. A reference electrode was placed on a bony prominence over the ankle.

The absolute values were taken prior to signal analysis. AcqKnowledge software Mean Power was calculated over 0.25 second epochs in a manually selected 4-second section (3 or 4 gait cycles). Epochs with greater than mean average Power were considered signal, all other epochs were considered noise. Signal to noise ratio (SNR) was calculated as the ratio between the average absolute signal EMG and the average absolute noise EMG.

The device eventually failed at week 12 , producing recordings with no clearly distinguishable EMG signals. Device failure was due to build up of debris inside the external socket connector (fig. 1(5)), effectively acting as an electrical insulator between plug and socket. 


\section{Impedence Spectroscopy}

In vitro impedance measurements were made using the EVAL-AD5934EBZ (Analog Devices, MA, USA) using the potentiostatic method [14].

The standard frequency range for the impedance monitor was $10^{3} \mathrm{~Hz}$ to $10^{5} \mathrm{~Hz}$ and the standard impedance range was $500 \Omega>Z>1 \mathrm{M} \Omega$. The impedance converter system was calibrated using a $100 \mathrm{k} \Omega$ (1\%) resistor before each measurement.

Impedance of pre-implanted and explanted epimysial electrodes were measured in $0.9 \%$ saline (physiological saline) at $1 \mathrm{kHz}$ to $77 \mathrm{kHz}, 0.4 \quad \mathrm{~V}_{\mathrm{p}-\mathrm{p}}$. The explanted electrode impedance was measured with $3 \mathrm{~mm}$ of surrounding tissue.

\section{E. Histological Evaluation}

After 12 weeks the animal was sacrificed and the implant removed with the electrode and a cuff of underlying muscle. Specimens were fixed in $10 \%$ formalin, dehydrated in an ascending alcohol series, defatted in chloroform, and embedded in LR White Resin (London Resin Company Limited, London, UK). Sections were cut, ground and polished to a thickness of $100 \mu \mathrm{m}$ and were then stained with toluidine blue. Histo-morphological assessment was carried out using an Olympus BH2 microscope (Olympus Optical Company Ltd, Tokyo, Japan), linked to Zeiss KS300 3.0 image-analysis software (Imaging Associates, Thame, UK).

\section{RESULTS}

\section{A. Gross morphology}

At 12 weeks the implant-skin interface was stable, showing no gross signs of infection or discharge (fig. 6.). A layer of

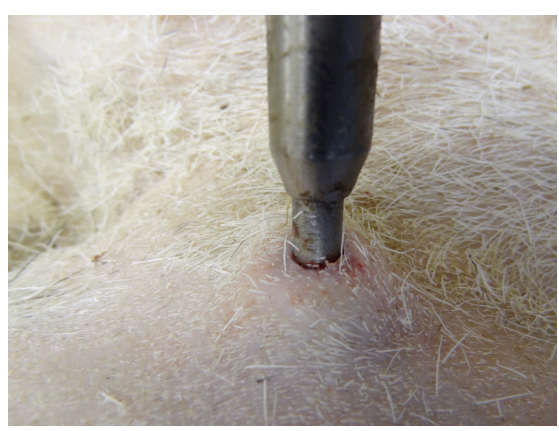

Fig. 6. Implant-skin interface at 12 weeks showing a stable skin interface.

fibrous tissue formed a capsule around the electrode and the electrode wire. There was no evidence of gross inflammation and the surrounding muscle appeared normal. The external socket connector showed heavy accumulation of debris within the electrical contact area effectively acting as an electrical insulator.

\section{B. Histological findings}

The silicone layer of the electrode was lost during grinding and polishing, leaving a distinct line at the interface with the underlying capsule. At 12 weeks the electrode interface

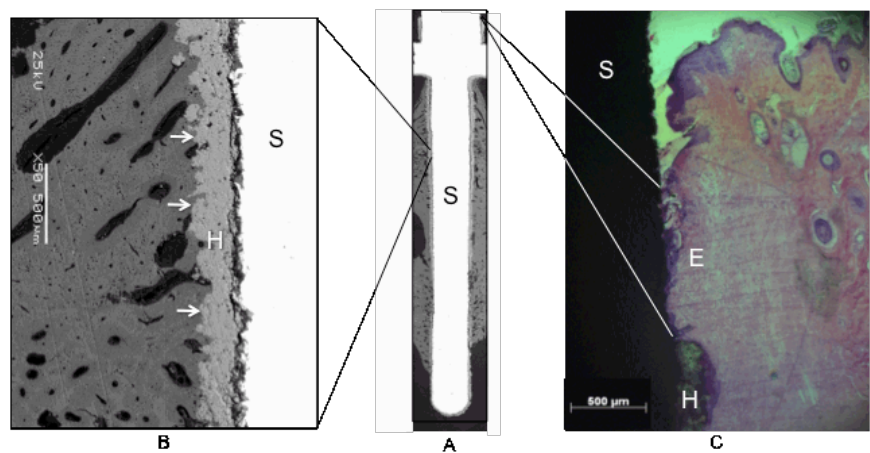

Fig. 7. A - Backscatter scanning electron micrograph of the boneanchored device. B - Arrows show 'osseointegration' with the HA coating (H) plasma sprayed onto the stem (S). C - Section through the skin-implant interface showing the keratinized epithelium (E) integrating with the stem (S) and adjacent $\mathrm{HA}$ coating $(\mathrm{H})$

showed encapsulation of fibrous tissue. The fibrous capsule was $250 \mu \mathrm{m}$ at its thickest. This consisted of parallel collagen fibres interspersed with fibroblasts. A well-demarcated layer of fibroblasts was seen at the interface with the electrode. Histological evaluation at the interface between the stem of the implant and the bone showed bone formation consistent with osseointegration. Epidermal and dermal attachment to the implant surface confirmed a stable soft-tissue-implant seal (fig. 7.).

\section{EMG results}

SNR tended to increase for the first 11 weeks of testing, from an initial value of 4.4 to a maximum of 6.5 in week 8

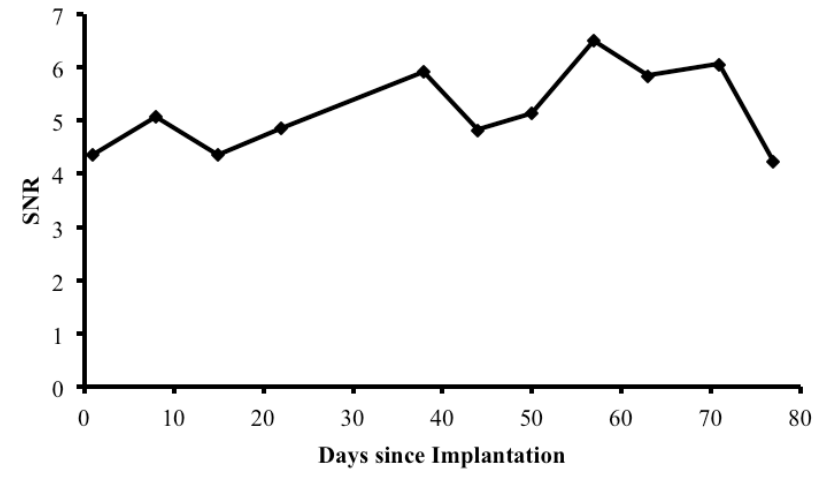

Fig. 8. SNR for epimysial electrode over the implantation period. Signal quality after the last reading shown deteriorated significantly due to build up of debris in the external socket compromising electric conduction.

(Fig. 8.). The SNR was greater for epimysial versus surface electrodes (5.1 and 1.6 respectively).

Signal quality deteriorated rapidly after 11 weeks and muscle activations became difficult to identify visually due to excessive noise. The $1 \mathrm{kHz}$ epimysial electrode impedance increased from $1.6 \mathrm{k} \Omega$ pre-implantation to $2.3 \mathrm{k} \Omega$ explanted.

\section{DISCUSSION}

This study is the first to combine EMG signal acquisition 
through a bone-anchored device from a cognisant, walking animal over 12-weeks. Throughout this period, the quality of the signals was maintained and the device eventually failed. This was due to build up of debris inside the exposed external socket connector (fig. 1(5)), a result of the sheep being housed in a barn environment. This was not due to soft-tissue issues. Fitting the external socket with a protective dust cap could have prevented this. The conduit and internal part of the electrode remained functional as demonstrated by impedance spectroscopy.

Implantable muscle electrodes have advantages over skinsurface electrodes: small, discreet or deep muscles responsible for specific actions can be individually targeted; electrode location is consistent and permanent; there are no effects of perspiration or patient weight-changes; and the impact of skin deformation is eliminated. Where TMR is indicated, the surgeon performing nerve coaptation can implant the electrodes during the same procedure, optimising accuracy of placement and eliminating the need to wear a waistcoat with a multitude of skin-surface electrodes [5]. By combining these advantages with the benefits of using bone-anchored prostheses, patients will have simpler, more robust systems with a single connection for both attachment and control.

Critical to the success of this approach is the establishment of a stable skin-implant interface. Traditional bone-anchored devices have suffered from a high rate of infection and chronic inflammation necessitating explantation [15], [16]. Pendegrass et al. [13] have demonstrated a bone-anchored device design with a robust skin-implant seal, which was clinically successful in a trans-humeral amputee [11]. A modification of this implant was used in our experiment.

Since the aim of the paper was to test the ability of the device to transmit EMG signals over time, we did not feel that an amputation model and longitudinal insertion was warranted. For this reason we implanted the device transversely. However, in human application, the device will be implanted longitudinally into the medullary canal of the long bone (radius or humerus).

In this model, we passed the electrode wire underneath the flange, removing the need for an additional hole in the cortex to transmit the wire, as described in a previous study [12]. As $\mathrm{HA}$ is osseoconductive, bone can grow along the surface of the stem right up to the flange area (fig. 7. A), a phenomenon known as bony bridging. However this did not affect transmission of signals. Furthermore, since the wire is encased in polymeric material, it does not osseointegrate. This setup simplifies the operative procedure and reduces the risk of wire damage when passing through an additional hole in the cortex (or potential damage during the bone-healing process).

Hard-wired constructs allow an increase in the number of channels without a disproportionate increase in the complexity of the implanted components. In contrast, signal telemetry requires ever increasing bandwidth, and therefore power consumption, to cope with high channel numbers and data rates. Crucially this system allows for a multi-channel setup providing the user with highly intuitive control of prosthesis.

Pitkin [12] reported on the use of a titanium pylon bone- anchored device as a conduit to take signals from internal microelectrodes through the skin barrier in a rabbit. The pylon was inserted into the tibia, and an additional hole was bored, through which wires exited the medullary canal. A single reading was obtained with the animal sedated by eliciting the 'righting reflex'. We recorded 'physiological' signals, by walking the animal on a treadmill, during a 12-week period. By passing the wire under the flange instead of the distal-most part of the bone-anchored device, we eliminated the need for an additional hole in the tibia.

The use of hard-wired bone-anchored devices is not limited to EMG signals. A sensory feedback loop could be setup transducing electrical impulses from pressure sensors in the prosthesis to sensory nerves in the residual stump. A similar setup can also be used for peripheral or central nervous system recordings and the recording of other biosignals. Direct recording of electroneurography (ENG) signals from peripheral motor nerves using implanted nerve electrodes can potentially yield all the signals required to control a prosthetic limb [1], [17]. The bandwidth requirements for ENG signals are greater than for EMG signals, and hard-wired transmission of these signals out of the body ensures fast data transfer without a drop in bandwidth associated with wireless telemetry. Accurate and reliable long-term ENG signal capture coupled with a bone-anchored device may provide the ideal solution for attachment and control of prosthetic limbs in the future.

An ovine model was chosen due to previous experience with bone-anchored devices in this model [13]. However, skin differences may make comparisons between epimysial and skin-surface EMG inapplicable to human patients. Additionally we have not evaluated potential signal interference from neighbouring muscles. To test this fully, selective intraoperative muscle stimulation can be used to measure cross-talk. To minimise cross-talk, the electrode was placed along the longitudinal axis of the muscle, with adjacent muscles (tibialis anterior and gastrocnemius) at least $20 \mathrm{~mm}$ from the center of the discs.

In addition to implantable electrodes, pattern recognition could provide a solution to simultaneous movements of the prosthesis, using several surface electrodes placed on the amputation stump to detect MES in the residual stump. Although studies have shown no significant difference in classification accuracy of pattern recognition-based myoelectric controllers utilising surface vs. intramuscular electrodes $[4,18]$, such experiments were carried out in the ideal laboratory environment, where problems related to the long-term consistency of signals were not fully investigated. These include signal changes due to skin impedance secondary to sweating, the need for repeated placements of electrodes, accuracy and consistency of electrode placement, electrode lift-off and motion artefact. These problems are addressed with implantable electrodes. Thus in systems employing direct control and pattern recognition, implantable electrodes may confer an advantage over surface electrodes.

Several design modifications are needed to translate this proof of concept into a human design, and work is underway 
to address these. The device will be inserted longitudinally into the medullary cavity of the radius/humerus instead of transcortically as described in this experiment. Additionally the seal around the borehole in the device will be a hermetic feed-through, to ensure a stable interface between the external and internal environments. The electrode wire will exit the device under the flange as described in this experiment, avoiding the need for an additional hole to be made in the diaphysis of the bone. Small alterations to the hard-wired system, such as the introduction of multiplexing, tripolar electrodes and electrode-site preamplification can be used to reduce the complexity of the implant-prosthesis connection, increase noise rejection, and reduce the noise resulting from cable motion respectively. Electrodes can be connected intraoperatively to the multiplexer using a reconnectable multiway implantable connector, which was shown to provide a stable long-term connection in human applications [18]. This setup allows individual replacement of electrodes/multiplexer should a fault arise in the future, without the need to replace the entire device.

The mechanical coupling between the device and the external prosthesis has been used by the co-author in an upper limb amputee fitted with a bone-anchored device, which will be applied to the final design. A fail-safe mechanism, which is designed to shear in half if the load applied to the prosthesis exceeds a specified limit, is inserted between the external part of the device and the external prosthesis. This also protects the bone from fracturing in the event of a fall onto the reconstructed arm [11].

A long-term, stable electrical coupling is currently under development with a similar failsafe device to protect the coupling in case of mechanical overloading.

This technology could also be applied to lower limb prostheses for control of ankle in below knee amputees with adequate residual leg muscles (tibialis anterior and gastrocnemius, which dorsiflex and plantarflex the ankle, respectively). However, the limited functional range of movement of the lower limb compared with the upper limb, and the availability of quality lower limb prostheses means the demand for an implantable electrode system is low.

\section{CONCLUSION}

1. This study was the first to combine EMG signal acquisition through a bone-anchored device from a cognisant, walking animal over a 12 -week period.

2. This technology forms the basis of a complete solution for upper limb amputees - both attachment and control.

3. Addition of a multiplexer allows a multichannel design, providing intuitive, physiological control of the end device.

\section{ACKNOWLEDGMENT}

Y. Al-Ajam thanks Gillian Hughes and Roberta Ferro de Godoy for technical assistance.

\section{REFERENCES}

[1] S. Micera, J. Carpaneto, and S. Raspopovic, "Control of Hand Prostheses Using Peripheral Information," Biomedical Engineering IEEE Reviews in, vol. 3, pp. 48 -68, 2010.

[2] K. Ziegler-Graham, E. J. MacKenzie, P. L. Ephraim, T. G. Travison, and R. Brookmeyer, "Estimating the prevalence of limb loss in the United States: 2005 to 2050," Arch Phys Med Rehabil, vol. 89, no. 3, pp. 422-429, Mar. 2008.

[3] S. A. Dalley, H. A. Varol, and M. Goldfarb, "A method for the control of multigrasp myoelectric prosthetic hands," IEEE Trans Neural Syst Rehabil Eng, vol. 20, no. 1, pp. 58-67, Jan. 2012.

[4] L. J. Hargrove, K. Englehart, and B. Hudgins, "A comparison of surface and intramuscular myoelectric signal classification," IEEE Trans Biomed Eng, vol. 54, no. 5, pp. 847-853, May 2007.

[5] T. A. Kuiken, G. Li, B. A. Lock, R. D. Lipschutz, L. A. Miller, K. A Stubblefield, and K. B. Englehart, "Targeted muscle reinnervation for real-time myoelectric control of multifunction artificial arms," JAMA, vol. 301, no. 6, pp. 619-628, Feb. 2009.

[6] R. F. ff Weir, P. R. Troyk, G. A. DeMichele, D. A. Kerns, J. F. Schorsch, and H. Maas, "Implantable myoelectric sensors (IMESs) for intramuscular electromyogram recording," IEEE Trans Biomed Eng, vol. 56, no. 1, pp. 159-171, Jan. 2009.

[7] M. Zecca, S. Micera, M. C. Carrozza, and P. Dario, "Control of multifunctional prosthetic hands by processing the electromyographic signal," Crit Rev Biomed Eng, vol. 30, no. 4-6, pp. 459-485, 2002.

[8] K. Warwick, M. Gasson, B. Hutt, I. Goodhew, P. Kyberd, B. Andrews, P. Teddy, and A. Shad, "The application of implant technology for cybernetic systems," Arch. Neurol., vol. 60, no. 10, pp. 1369-1373, Oct. 2003

[9] D. Dumitru, J. C. King, and W. E. Rogers, "Motor unit action potential components and physiologic duration," Muscle Nerve, vol. 22, no. 6, pp. 733-741, Jun. 1999.

[10] I. Dudkiewicz, R. Gabrielov, I. Seiv-Ner, G. Zelig, and M. Heim, "Evaluation of prosthetic usage in upper limb amputees," Disabil Rehabil, vol. 26, no. 1, pp. 60-63, Jan. 2004.

[11] N. V. Kang, C. Pendegrass, L. Marks, and G. Blunn, "Osseocutaneous integration of an intraosseous transcutaneous amputation prosthesis implant used for reconstruction of a transhumeral amputee: case report," J Hand Surg Am, vol. 35, no. 7 pp. 1130-1134, Jul. 2010.

[12] M. Pitkin, C. Cassidy, R. Muppavarapu, and D. Edell, "Recording of electric signal passing through a pylon in direct skeletal attachment of leg prostheses with neuromuscular control," IEEE Trans Biomed Eng, vol. 59, no. 5, pp. 1349-1353, May 2012.

[13] C. J. Pendegrass, A. E. Goodship, and G. W. Blunn, "Development of a soft tissue seal around bone-anchored transcutaneous amputation prostheses," Biomaterials, vol. 27, no. 23, pp. 4183-4191, Aug. 2006.

[14] A. Prasad and J. C. Sanchez, "Quantifying long-term microelectrode array functionality using chronic in vivo impedance testing," J Neural Eng, vol. 9, no. 2, p. 026028, Apr. 2012.

[15] J. Sullivan, M. Uden, K. P. Robinson, and S. Sooriakumaran, "Rehabilitation of the trans-femoral amputee with an osseointegrated prosthesis: the United Kingdom experience," Prosthet Orthot Int, vol. 27, no. 2, pp. 114-120, Aug. 2003.

[16] S. Sooriakumaran, K. P. Robinson, and D. A. Ward, "Pattern of infection of transfemoral osseointegration. In: International society for prosthetics and orthotics 11th world congress. Hong Kong. 2004."

[17] X. Navarro, T. B. Krueger, N. Lago, S. Micera, T. Stieglitz, and P. Dario, "A critical review of interfaces with the peripheral nervous system for the control of neuroprostheses and hybrid bionic systems," J. Peripher. Nerv. Syst., vol. 10, no. 3, pp. 229-258, Sep. 2005.

[18] D. N. Rushton, A. M. Tromans, and N. de N. Donaldson, "A reconnectable multiway implantable connector," Med Eng Phys, vol. 24, no. 10, pp. 691-694, Dec. 2002. 